\title{
THE TWO MICRON ALL SKY SURVEY
}

\section{Survey Strategy and Prototyping}

S. G. KLEINMANN, M. G. LYSAGHT, W. L. PUGHE, S. E. SCHNEIDER, M. F. SKRUTSKIE and M. D. WEINBERG

University of Massachusetts

S. D. PRICE

Air Force Phillips Laboratory

K. Y. MATTHEWS and B. T. SOIFER

California Institute of Technology

J. P. HUCHRA

Center for Astrophysics

C. A. BEICHMAN, T. J. CHESTER, T. JARRETT, G. L. KOPAN and C. J. LONSDALE

Infrared Processing and Analysis Center

J. ELIAS

National Optical Astronomy Observatories

J. W. LIEBERT

University of Arizona

and

P. SEITZER

University of Michigan

\begin{abstract}
This paper describes the design, expectations, and prototyping of a new allsky survey, called 2MASS (Two Micron All Sky Survey) to be carried out with the new generation of infrared array detectors.
\end{abstract}

Key words: sky survey - infrared star counts - infrared galaxy counts

\section{Introduction}

The 2MASS (Two Micron All Sky Survey) project is an effort to obtain a uniform map of the whole sky in the near-infrared. The survey will be executed simultaneously in 3 wavebands, centered at $1.25(\mathrm{~J}), 1.65(\mathrm{H})$, and $2.16\left(\mathrm{~K}_{s}\right) \mu \mathrm{m}$, with a pixel size of $2.3^{\prime \prime} \times 2.3^{\prime \prime}$. It will have sufficient sensitivity to measure accurate positions and brightnesses for $\sim 100$ million stars and $\sim 1$ million galaxies. This survey would not be possible without the new generation of infrared-sensitive array detectors; at the same time, it provides a database that is critically needed in order to apply those arrays to interesting and objectively selected sources to study.

The only existing large-area near-infrared sky survey, the Two Micron Sky Survey (Neugebauer and Leighton 1969; hereafter TMSS), reached a 

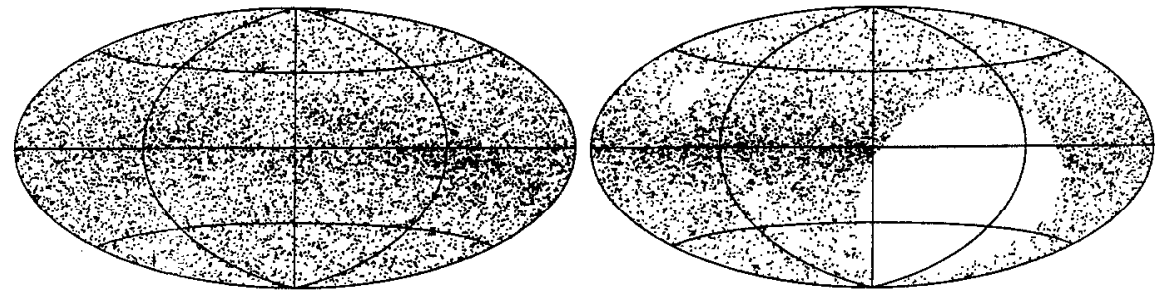

Fig. 1. The distribution of stars in galactic coordinates for (left) the Yale Bright Star Catalog and (right) the Two Micron Sky Survey.

statistical limit of $K=+3$ mag., sufficient to detect about 5600 stars over the region surveyed, or about $0.20 \mathrm{stars} / \mathrm{deg}^{2}$. This surface density is comparable to that of the Bright Star Catalog (Hoffleit and Jaschek 1982; hereafter BSC); but a comparison of the apparent space distributions of the TMSS and BSC (Figure 1) shows that the whole sky looks different in the nearinfrared.

This is partly due to the fact that the interstellar medium is far more transparent in the near-infrared than the optical $\left(A_{K} \simeq 0.10 A_{V}\right)$, and partly due to the fact that a different population of stars is being detected in the near-infrared: over half of the stars in the BSC are dwarfs within $\sim 200 \mathrm{pc}$, whereas $98 \%$ of the stars in the TMSS are evolved giant stars at distances up to $\sim 1.5 \mathrm{kpc}$.

2MASS will see evolved stars throughout the entire disk of the Milky Way, and will detect other galaxies (by virtue of their giant stars) out to distances well beyond the range in which local motions dominate the Hubble flow. The 2MASS project was designed to provide the sensitivity, uniformity, and sky coverage that would enable its use in studies of the large-scale structure of the Milky Way and the Local Universe.

Designation of the optimum design parameters for a survey of this sensitivity was initially hindered by a significant number of unknowns. For example, at the start of this project, only rough estimates were available for such key parameters as the source densities in sparse regions (e.g., the North Galactic Pole), or the region of the sky where the survey would be confusion limited. Also, little information was available on the luminosities, shapes, and other properties of galaxies that would be brightest in the nearinfrared, or the variation of skyglow emission with season, time of night, elevation, and moon phase and angle. Added to these questions were large practical problems, including the need to develop a realistic solution to the 
mechanical requirements of covering such a large region of sky in $\lesssim 2$ years, and the need to demonstrate the feasibility of processing a data base that was expected to be $\sim 1000 \times$ larger than the IRAS data base.

To address these questions, a prototype camera was built and has been used at the Kitt Peak National Observatory. This paper describes our use of that camera ( $\$ 2$ ), including an initial assessment of the quality of results. From observations of over $800 \mathrm{sq}$. deg. to date, we have measured star counts over a wide range in galactic latitude and longitude (\$3), as well as galaxy counts in selected regions ( $\$ 4)$. We conclude $(\$ 5)$ by summarizing our plans for implementing the full survey and providing the data to the community.

\section{The Prototype Camera}

The prototype camera was built at Infrared Laboratories (Tucson, AZ) during the first 4 months of 1992. It contains a single NICMOS3 (HgCdTe; $256 \times 256$ ) science grade array from Rockwell International Science Center, mounted in an $\mathrm{LN}_{2}$ dewar behind a cooled, manually-controlled filter wheel which provides a means of mapping at any one of the survey wavebands.

The camera was mounted on the Kitt Peak $1.3 \mathrm{~m}$ telescope. Mapping was accomplished by using a technique dubbed "freeze frame scanning," whereby the telescope tracks in Right Ascension, and scans in declination, while the secondary mirror is driven in a sawtooth pattern which freezes an image on the focal plane for the long part of the sawtooth. The array was read out twice at each position, once corresponding to just $(51 \mathrm{~ms})$ after reset, and once $1.5 \mathrm{~s}$ later. Use of these two reads extends the dynamic range of the survey, allowing accurate photometry of stars as bright as $K_{s}=4 \mathrm{mag}$.

The camera has been used with a science grade array on 5 observing runs at Kitt Peak since May 1992. These included $\sim 20$ photometric nights. Data obtained from these runs have been processed at JPL's Infrared Processing and Analysis Center. The processing pipeline is itself a prototype, but has allowed a demonstration of the feasibility of the data processing task, and has provided a baseline from which one could judge the quality of the survey data.

The point spread function produced by the scanning technique is shown in Fig. 2. The major image broadening in the current configuration is due to chromatic aberration.

The quality of the data was tested by repeated observations through M92; briefly, the $1 \sigma$ scatter in the photometry is $5 \%$ for $8 \leq K_{s} \leq 13$ mag. The repeatability of the positions of extracted sources has a dispersion of $\leq 0.3^{\prime \prime}$ for $K_{s} \leq 14$ mag. 


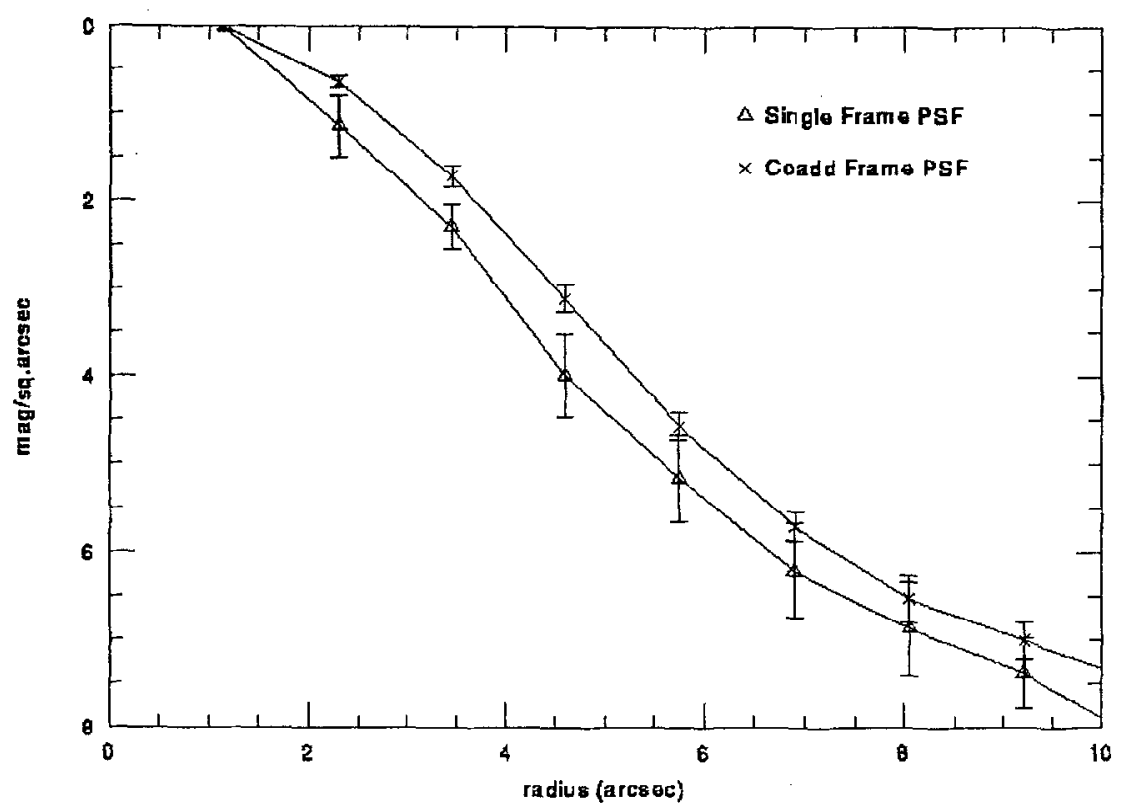

Fig. 2. The point spread function in the combination of 5 frames and the PSF extracted from the individual frames. The average PSF is much more stable than the undersampled data in any one frame and leads to accurate photometry. The average PSF is broadened in the prototype camera due to variations of the PSF across the focal plane.

\section{Star Counts}

Star counts in the regions observed to date, within the longitude range, $40^{\circ}<l<60^{\circ}$, are shown in Figure 3. The data in the figure are compared to a simple model that incorporates only an exponential disk following Bahcall and Soneira (1981). The model also incorporates reddening due to an isothermal disk with a scale height of $100 \mathrm{pc}$ and a reddening gradient of $\Delta \mathrm{K}_{s}=0.18 \mathrm{mag} . / \mathrm{kpc}$. This simple model provides an acceptable fit in the range $0^{\circ}<b<90^{\circ}$ over a wide range of longitudes.

A more detailed example of the fit of the data to integrated star counts from $5<K_{s}<14$ mag. is shown in Figure 4. for a 1 sq. deg. region near $1=53^{\circ}, b=0^{\circ}$. The quality of the fit is essentially the same in all regions analyzed to date. Thus, a simple exponential disk model which includes reddening appears to be a good first-order means of estimating the "background" star counts, over latitudes in the range $0^{\circ}<b<90^{\circ}$, longitudes in the range $25^{\circ}<l<160^{\circ}$, and stars in the range $\mathrm{K}_{\mathrm{s}}<14$ mag.

Studies of the large-scale structure of the galactic disk using 2MASS results will rely most heavily on luminous giant branch stars, because of 


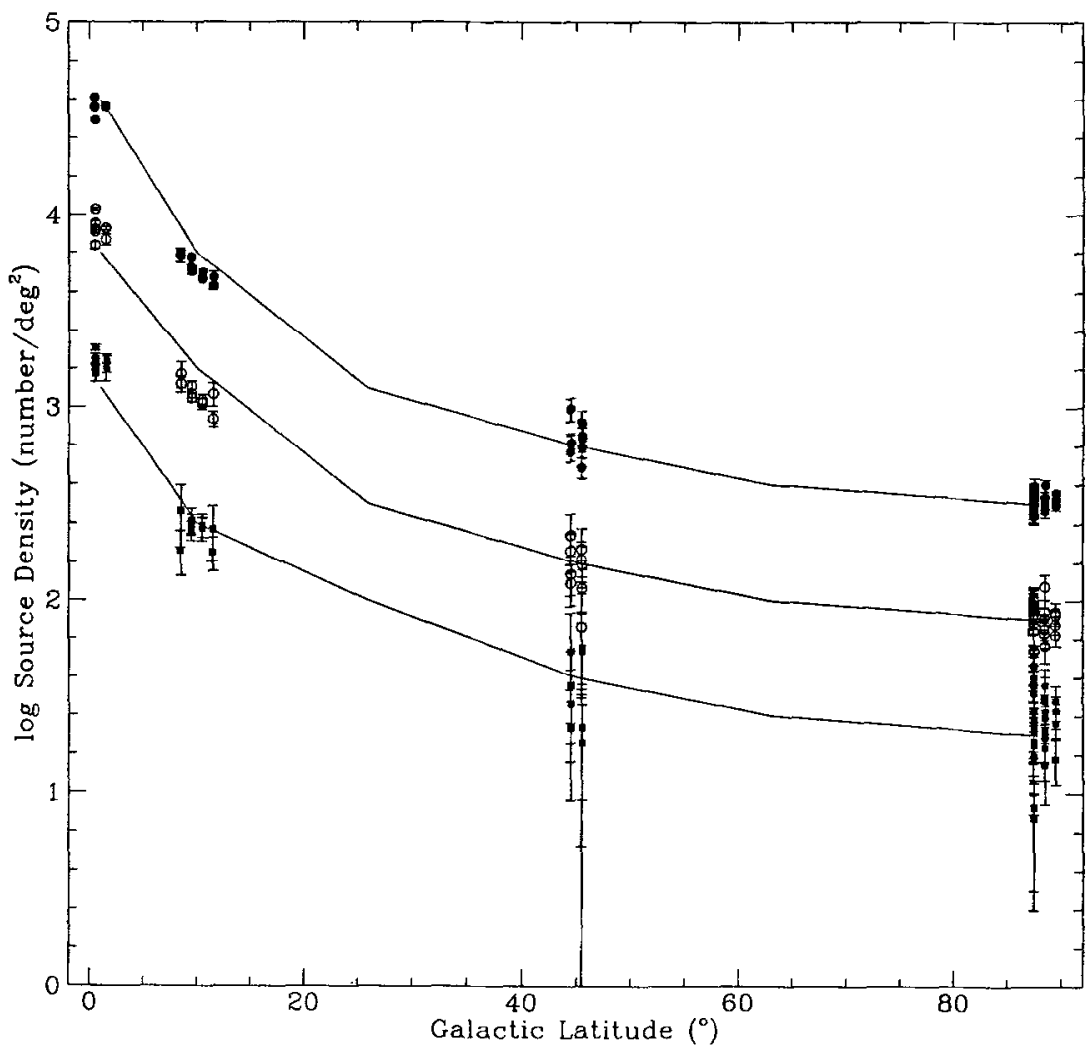

Fig. 3. The integrated surface density number of sources brighter than $K_{s}=10$ (filled triangles), 12 (open circles), and 14 (filled circles) as a function of galactic latitude for the longitude range $40^{\circ} \leq l \leq 60^{\circ}$. The curves show the expected distribution for a model incorporating a simple exponential disk with reddening, and including AGB stars (see text).

their ubiquity, luminosity, and age. Most of these stars will be detected at $\mathrm{K}_{s}<10$ mag. because at fainter levels such stars would be seen at or beyond the edge of the disk. Accurate photometry of such bright stars is obtained by exploiting the fact that the arrays can be read non-destructively, and executing the first of two readouts of the array almost immediately after reset. The first readout provides a measurement with an integration time nearly $30 \times$ shorter than the second readout, and thereby extends the dynamic range by $\geq 3.5$ mag. Comparisons of the photometry obtained with this technique to photometry of the same stars measured with a single element InSb photometer at Whately Observatory, show that the rms errors in the 2MASS read-after-reset photometry is $\leq 5 \%$ for stars as bright as $\mathrm{K}_{s}$ $=4$ mag. 


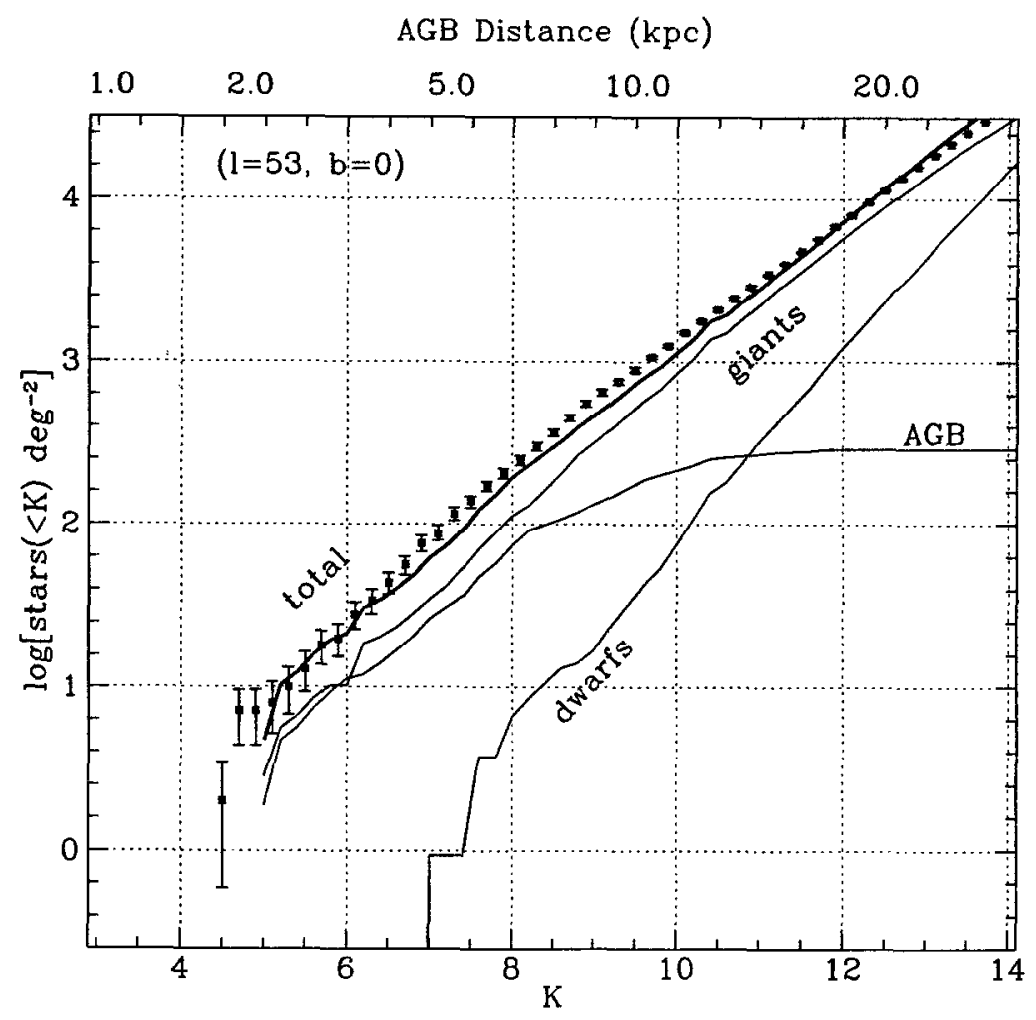

Fig. 4. Expected cumulative count density (number per square degree) in a field at Galactic coordinates $l=53^{\circ}, b=0^{\circ}$ together with the prototype camera results (solid squares). The contributions of counts from dwarfs, giants and AGB stars are also separately shown.

\section{Galaxy Counts}

Galaxies are located within the prototype camera image database by seeking objects with surface brightness gradients smaller than those of stars. (Note that this contrasts sharply with the IRAS experience, where galaxies could be distinguished by their non-stellar colors.) Since this exercise is hazardous in an undersampled image, particularly one close to the Galactic plane, we observed selected regions at middle and high Galactic latitudes to obtain a first assessment of the number and properties of galaxies that could be seen in the survey. Most of these observations were carried out at $K_{s}$, because the current optics configuration of the prototype camera provides best performance at that wavelength.

The field most intensively analyzed to date is centered near $\left(l=135^{\circ}\right.$, $b=-25^{\circ}$ ), which covers about 11 sq. deg. 283 galaxies were detected to 


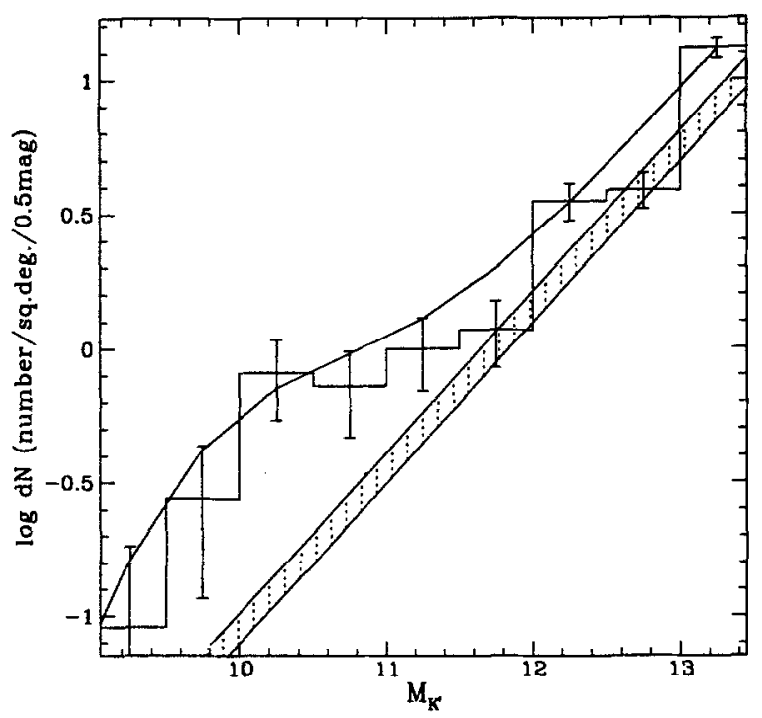

Fig. 5. The surface density of galaxies in the $\left(l=135^{\circ}, b=-25^{\circ}\right)$ field (histogram) compared with surface densities of field galaxies from Broadhurst et al. 1992 (shaded line). The large excess of galaxies at $K,<11 \mathrm{mag}$. appears to be caused by the large number of galaxies concentrated at $z \sim 0.017$. A model of field counts plus supercluster members (solid line) is described in the text.

$\mathrm{K}_{s} \leq 13.5$ which is 5 times more than the number found by Zwicky et al. (1961-8) down to that catalog's completeness limit of $m_{B} \sim 15.5$ mag. Extrapolating these counts and those from other fields against the total counts and coverage of the $\mathrm{Zwicky}$ catalog, and accounting for the effects of Galactic obscuration, we estimate that $\sim 500,000$ galaxies will be identified at $\mathrm{K}$-band over the whole sky. The J-band census of galaxies will be even richer.

The $\left(l=135^{\circ}, b=-25^{\circ}\right)$ field is located in the region of the PiscesPerseus supercluster, where there is a strong concentration of galaxies at redshifts $z \sim 0.017$. The differential source counts are shown in Figure 5 and are compared with galaxy counts from Broadhurst et al. (1992) in selected fields. Due to the excess of galaxies at $z \sim 0.017$, and because of the shape of the galaxy luminosity function, we expect an excess of galaxies near the apparent magnitude of an $L_{*}$ galaxy at that redshift. As a model of the contribution from the supercluster galaxies, we show the effect of adding excess galaxies at $z \sim 0.017$. The galaxies are assumed to obey a Schechter 
(1976) luminosity function, with parameters for K-band from Cowie et al. (1992): $\phi_{*}=2.3 \times 10^{-3} \mathrm{Mpc}^{-3}, M_{*}(K)=-25$, and $\alpha=-1.1$. The difference between the 2MASS galaxy counts in this region and those found in pencil beam surveys emphasizes the impact of "local large scale structure" on galaxy counts, and the importance of measuring the space distribution of galaxies over many regions of the sky-preferably the whole sky.

\section{Implementation}

The survey will be carried out on two matched telescopes to be sited at Mt. Hopkins and Cerro Tololo. Operations are expected to begin in 1996, and be completed in $\sim 2$ years.

2MASS results will be made available to the community as soon as they pass verification tests by the science team; no proprietary period has been included in the schedule. It is expected that data distribution will begin within 1.5 years of the start of the survey. The data products will include point and extended source catalogs, and an image atlas; the entire database of raw images will also be available to the community.

\section{Acknowledgements}

Frank Low and Fred Gillett contributed greatly to the initial development of this project, especially the implementation of the prototype camera at Kitt Peak. R. M. Light (IPAC) did the analysis for Figure 2. This project has been supported by the Air Force Phillips Laboratory, NASA, NSF, the United States Navy, and the University of Massachusetts.

\section{References}

Bahcall, J. N., and Soneira, R. M.: 1981, Astrophysical Journal, Supplement Series 47, 357

Broadhurst, T. J., Ellis, R. S., and Glazebrook, K.: 1992, Nature 355, 55

Cowie, L. L., Gardner, J. P., Hu, E. M., Wainscoat, R. J., and Hodapp, K. W.: 1993, Astrophysical Journal, submitted

Hoffleit, D., with the collaboration of Jaschek, C.: 1982, The Yale Bright Star Catalog, 4th rev. ed., Yale University Press: New Haven, CN

Neugebauer, G. and Leighton, R. B.: 1969, The Two Micron Sky Survey, U. S. Government Printing Office: Washington, D. C.

Schechter, P.: 1976, Astrophysical Journal 203, 297

Zwicky, F., Herzog, E., Wild, P., Karpowics, M., and Kowal, C. T.: 1961-1968, Catalog of Galaxies and Clusters of Galaxies, Volumes I-VI, California Institute of Technology: Pasadena 